\title{
Gap Analysis between Theory and Practice of Software Testing Methodologies in Indian IT Industry
}

\author{
Dilbag Singh, Ph.D \\ Dept. of CSA \\ Ch. Devi Lal University, Sirsa
}

\author{
Pradeep Kumar, Ph.D \\ Dept. of IT \\ LBS Inst. of Mgmt., Delhi
}

\author{
Sanjeev Kumar \\ Dept. of CSA \\ Ch. Devi Lal University, Sirsa
}

\begin{abstract}
There are broadly two approaches for software testingmanually or automatically. Manual testing uses the knowledge of the tester to target testing of the system that is assumed to be more error-prone. Automated testing can perform a large number of tests in little time. Software testing is taught to the computer/IT graduates in Indian universities. Tools for manual and automatic testing are available in the market to enhance the productivity and reliability of the testing process.

The survey focused on three major aspects of software testing, namely software testing education/ training, testing methodologies/ techniques, and automated testing tools. Based on the survey results, current practices in software testing are reported, as well as some observations and recommendations for the future of software testing in India for academia and industry.
\end{abstract}

Keywords: Software testing, Manual testing, Automated testing, IT Industry

\section{INTRODUCTION}

Software testing is the process of executing a program with the intention of finding errors in the code [4]. It is the process of exercising or evaluating a system or system component by manual or automatic means to verify that it satisfies specified requirements or to identify differences between expected and actual results [8]. Software Testing should not be a distinct phase in system development but should be applicable throughout the design development and maintenance phases. Software testing is the process of executing software in a controlled manner, in order to answer the question. Does the software behave as specified? One way to ensure system's responsibility is to extensively test the system [9]. Since software is a system component it requires a testing process also. The overall testing process benefits from the strengths of both manual and automated testing [10].

With a manual strategy, testers prepare a test suite that generates test cases from the program's specification (black box) or its actual text (white box) [7]. An automated testing strategy tries to remove the tediousness of the process by relying on a software tool. Automated and manual strategies are often thought of as completely distinct, and usually supported by different tools. Manual tests are good for capturing deep or special cases, which automated tests might not guess [11]. But they cannot yield extensive coverage. Automated tests are good at breadth but much less at depth. Manual unit testing has established itself as an integral part in modern software development [12]. Automated testing automates not only test case execution, but also test case generation and test result verification. A fully automated testing system is able to test software without any user intervention [1]. Automated testing requires less effort on the developer's side, but it cannot fully replace manual unit testing. Developers are better at setting up complex input data and at finding interesting test cases [2].

There were a number of reasons for conducting this survey: Firstly, to determine whether existing training courses in software testing taught in the workplace or in similar study at Indian universities adequately cover the types of testing methodologies and skills that industry requires. Secondly, to identify gap between theory and practices for both manual and automated testing in IT industry. Finally, the survey may provide indications of future research directions. The observations reported in this paper are based on 107 respondents for manual testing and 104 respondents for automation testing who have completed the questionnaire successfully. The respondents were from various IT companies from NCR region. Despite the relatively small sample population in the survey, the consistency of the data obtained heightened our confidence to report the observations in this paper.

\section{METHOD DESCRIPTION}

\subsection{Survey Description}

The survey targeted senior employees involved with testing in software development organizations. Requests were addressed to software testing or quality mangers of the IT companies in NCR region to understand their testing environments and experiences.

Three major areas of software testing related activities like software testing education/ training, software testing methodologies/techniques, automated software testing tools etc. were investigated by the survey. The information sought can be summarized as follows [5].

Software Testing Education/ Training: It determined the extent to which they have learnt the knowledge of softer testing through academics and training by the organizations for their employees. The usages of various sources of training courses were also queried [3].

Software Testing Methodologies/Techniques: The extent to which software testing methodologies and general testing techniques are used in the industry and the current practices of those organizations adopting structured methodologies and techniques in software testing were investigated [1].

Automated Software Testing Tools: Questions relating to the extent to which automated testing tools are used in industry, including commercial and in-house developed tools, were revealed. The level of satisfaction with such 
tools was assessed by querying the respondents' belief that the quality of developed software was being improved by the use of such tools [6].

\subsection{Survey Method}

A questionnaire comprised 25 questions in each of manual and automated testing was used. Survey interviews were conducted face to face, over the telephone, via email attachment. To allow for more flexible arrangements, some respondents were invited to complete the online questionnaire at our survey website. In all cases, printed or verbal explanatory notes were provided to respondents to ensure consistent interpretation of the terminologies and questions in the questionnaire. Confidentiality and privacy were assured to all individuals returning the questionnaire and the organization that they represented.

\subsection{Sample Selection and Responses}

\subsubsection{Sample Selection}

Our survey targeted the population at the organizational level. A draft questionnaire of the survey was trialed against a small group of organizations, and a number of adjustments were made based on the experiences and feedback we gathered from respondents. As a result, we aimed at targeting four different types of participants in this survey. The first preference was test managers, the second was a member of the test team, thirdly a software quality manager, and finally a general IT professional having testing experience. This allowed us to deal with situations where there was no specific individual responsible for testing in the organization.

The both questionnaire consists of five-level Likert-scale response alternatives: Strongly disagree, Disagree, Neutral, Agree, and Strongly Agree. As a result, 107 respondents for manual testing and 104 respondents for automation testing completed the questionnaire successfully in the survey. This is a relatively low response rate, given the large number of organizations that were invited to participate in the survey, and the large estimated size of the population. The questionnaires were pre-tested. The purpose was to test the instruments for validity \& reliability to determine how realistic the questions were to the ability of users. Minor changes were made after the pre-test, based on feedback we gathered from respondents. Cronbach's Alpha test was used on the data. It provides a measure of the internal consistency of a test or scale. It is expressed as a number between 0 and 1 . Value of alpha for manual testing was .683 and for automated testing it was .717 calculated using SPSS software.

\subsubsection{Responses}

The responses of survey on manual testing are provided in table- 1 and responses of survey on automated testing are provided in table- 2 .

\section{ANALYSIS OF RESULT}

\subsection{Gap Analysis of Theory and Practice for Manual Testing}

Data of 107 respondents was analyzed by applying factor analysis using SPSS software. The purpose of factor analysis is to reduce multiple variables to a lesser number of underlying factors that are being measured by the variables. To test the appropriateness of factor analysis technique Kaiser-Meyer-Olkin (KMO) measure of sample adequacy is used. KMO compares the magnitude of observed correlation coefficients to magnitude of partial correlation coefficients. KMO identified values greater than 0.5 might desirable. In our analysis, the value of KMO comes out is .727 with significance .000 , which is acceptable.

Principal Component Analysis is a dimension reduction technique to analyze factors responsible for gap in manual software testing. A set of factors is identified based upon possible information available in the data. Nine factors are extracted on the basis of Principal Component Analysis. These nine factors describe the relationship among variables in a best way showing cumulative \%age of variances, it can be observed that nine factors identifying $71.192 \%$ of variance contributed by first component is 19.482 followed by second (27.486), third (34.793), fourth (41.091), fifth (47.314), sixth (53.467), seventh (59.598), eighth (65.601) and last ninth is (71.192) [Table-3]. In rotated component matrix, a value greater than .500 is considered for analysis across these nine factors against 25 question statements

\subsubsection{Manual Testing Factors [Table-4]:}

Factor 1: In factor 1, four variables found to be high factor loading i.e. .801 (Final project training is actually done at $\mathrm{s} / \mathrm{w}$ industry in testing domain), .505 (Manual s/w testing concepts used in industry are same as you studied), .832(For manual testing job, training is not required from industry /private institute), and .847 (In house training is not required for manual testing). So these four variables can be clubbed and factor may be named as "There is no gap between theoretical and actual implementation of manual s/w testing".

Factor 2: In factor 2, two variables found to be high factor loading i.e. .889(Implementation (Practical) of software testing strategies was part of curriculum), and .666(Marks of implementation (practical's) of ST added to marks of final degree). So these two variables can be clubbed and factor may be named as "Software testing syllabus includes practical of manual testing techniques and marks are added to degree".

Factor 3: In factor 3, two variables found to be high factor loading i.e. .847 (Implementation (Practical) of software engineering concepts was part of curriculum), and .839 (Marks of implementation (practical) of SE added to marks of final degree). So these two variables can be clubbed and factor may be named as "Software engineering practical are not adequate in curriculum but marks are added to degree".

Factor 4: In factor 4, two variables found to be high factor loading i.e. .736(Academic curriculum covers in depth knowledge of Software engineering (SE)), and .708(SE curriculum covers architectural design of software). So these two variables can be clubbed and factor may be named as "Software engineering has theoretical foundation in curriculum".

Factor 5: In factor 5, two variables found to be high factor loading i.e. .686(SE syllabus covers basic knowledge of software testing), and .660(ST was a separate subject with focus on manual testing in academic curriculum). So these two variables can be clubbed and factor may be named as "Software testing has theoretical foundation in curriculum" 
Table-1: Responses of survey on manual testing

\begin{tabular}{|c|c|c|c|c|c|c|c|c|c|c|c|}
\hline & \multirow[t]{2}{*}{ Questions } & \multicolumn{2}{|c|}{$\begin{array}{l}\text { Strongly } \\
\text { Disagree }\end{array}$} & \multicolumn{2}{|c|}{ Disagree } & \multicolumn{2}{|c|}{ Neutral } & \multicolumn{2}{|c|}{ Agree } & \multicolumn{2}{|c|}{$\begin{array}{c}\text { Strongly } \\
\text { Agree }\end{array}$} \\
\hline & & 总 & $\%$ & 导 & $\%$ & 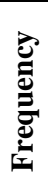 & $\%$ & 导 & $\%$ & 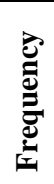 & $\%$ \\
\hline 1 & $\begin{array}{l}\text { Academic curriculum provides only basic } \\
\text { knowledge of Software testing (ST). }\end{array}$ & 10 & 9.3 & 61 & 57.0 & 15 & 14.0 & 15 & 14.0 & 6 & 5.6 \\
\hline 2 & $\begin{array}{l}\text { Academic curriculum covers in depth knowledge of } \\
\text { Software engineering (SE). }\end{array}$ & 5 & 4.7 & 6 & 5.6 & 22 & 20.6 & 58 & 54.2 & 16 & 15.0 \\
\hline 3 & $\begin{array}{l}\text { Implementation (Practicals) of software engineering } \\
\text { concepts was part of curriculum. }\end{array}$ & 11 & 10.3 & 32 & 29.9 & 22 & 20.6 & 35 & 32.7 & 7 & 6.5 \\
\hline 4 & $\begin{array}{l}\text { Marks of implementation (practical) of SE added to } \\
\text { marks of final degree. }\end{array}$ & 8 & 7.5 & 29 & 27.1 & 28 & 26.2 & 35 & 32.7 & 7 & 6.5 \\
\hline 5 & $\begin{array}{l}\text { SE syllabus covers basic knowledge of software } \\
\text { testing. }\end{array}$ & 5 & 4.7 & 5 & 4.7 & 5 & 4.7 & 47 & 43.9 & 45 & 42.1 \\
\hline 6 & $\begin{array}{l}\text { Academic curriculum of } \mathrm{SE} \text { meets the } \\
\text { requirements of present s/w industry }\end{array}$ & 3 & 2.8 & 4 & 3.7 & 14 & 13.1 & 43 & 40.2 & 43 & 40.2 \\
\hline 7 & $\begin{array}{l}\text { ST was a separate subject with focus on manual } \\
\text { testing in academic curriculum. }\end{array}$ & 2 & 1.9 & 1 & 0.9 & 14 & 13.1 & 47 & 43.9 & 43 & 40.2 \\
\hline 8 & $\begin{array}{l}\text { ST syllabus provided knowledge of black box } \\
\text { testing. }\end{array}$ & 8 & 7.5 & 19 & 17.8 & 21 & 19.6 & 38 & 35.5 & 21 & 19.6 \\
\hline 9 & $\begin{array}{l}\text { ST syllabus provides knowledge of white box } \\
\text { testing. }\end{array}$ & 6 & 5.6 & 22 & 20.6 & 23 & 21.5 & 37 & 34.6 & 19 & 17.8 \\
\hline 10 & $\begin{array}{l}\text { In depth knowledge of complete software testing } \\
\text { was part of curriculum. }\end{array}$ & 4 & 3.7 & 1 & 0.9 & 21 & 19.6 & 45 & 42.1 & 36 & 33.6 \\
\hline 11 & $\begin{array}{l}\text { Final project training is actually done at } \mathrm{s} / \mathrm{w} \\
\text { industry in testing domain }\end{array}$ & 14 & 13.1 & 26 & 24.3 & 22 & 20.6 & 25 & 23.4 & 20 & 18.7 \\
\hline 12 & $\begin{array}{l}\text { Project classes and practical viva was under control } \\
\text { of industry expert. }\end{array}$ & 5 & 4.7 & 13 & 12.1 & 13 & 12.1 & 44 & 41.1 & 32 & 29.9 \\
\hline 13 & $\begin{array}{l}\text { Implementation (Practical) of software testing } \\
\text { strategies was part of curriculum. }\end{array}$ & 6 & 5.6 & 25 & 23.4 & 27 & 25.2 & 36 & 33.6 & 13 & 12.1 \\
\hline 14 & $\begin{array}{l}\text { SE curriculum covers architectural design of } \\
\text { software. }\end{array}$ & 4 & 3.7 & 5 & 4.7 & 6 & 5.6 & 52 & 48.6 & 40 & 37.4 \\
\hline 15 & $\begin{array}{l}\text { Regression testing is part of theory/software } \\
\text { maintenance. }\end{array}$ & 5 & 4.7 & 5 & 4.7 & 5 & 4.7 & 50 & 46.7 & 42 & 39.3 \\
\hline 16 & $\begin{array}{l}\text { Manual s/w testing concepts used in industry are } \\
\text { same as you studied. }\end{array}$ & 10 & 9.3 & 14 & 13.1 & 16 & 15.0 & 41 & 38.3 & 26 & 24.3 \\
\hline 17 & $\begin{array}{l}\text { More emphasis on programming languages than SE } \\
\text { in curriculum }\end{array}$ & 5 & 4.7 & 17 & 15.9 & 21 & 19.6 & 43 & 40.2 & 21 & 19.6 \\
\hline 18 & $\begin{array}{l}\text { Live projects of ST were included in curriculum } \\
\text { through industry collaboration. }\end{array}$ & 22 & 20.6 & 54 & 50.5 & 20 & 18.7 & 10 & 9.3 & 1 & 0.9 \\
\hline 19 & $\begin{array}{l}\text { Implementation (Practical) of software testing } \\
\text { strategies should be part of curriculum. }\end{array}$ & 12 & 11.2 & 29 & 27.1 & 7 & 6.5 & 29 & 27.1 & 30 & 28.0 \\
\hline 20 & $\begin{array}{l}\text { Marks of implementation (practical's) of ST added } \\
\text { to marks of final degree. }\end{array}$ & 10 & 9.3 & 14 & 13.1 & 26 & 24.3 & 35 & 32.7 & 22 & 20.6 \\
\hline 21 & $\begin{array}{l}\text { Able to join software testing job directly after } \\
\text { completion of academics. }\end{array}$ & 14 & 13.1 & 33 & 30.8 & 32 & 29.9 & 21 & 19.6 & 7 & 6.5 \\
\hline 22 & $\begin{array}{l}\text { ST syllabus provides knowledge of object oriented } \\
\text { testing. }\end{array}$ & 3 & 2.8 & 8 & 7.5 & 19 & 17.8 & 45 & 42.1 & 32 & 29.9 \\
\hline 23 & $\begin{array}{l}\text { SE concepts used in industry are same as you } \\
\text { studied. }\end{array}$ & 4 & 3.7 & 3 & 2.8 & 21 & 19.6 & 41 & 38.3 & 38 & 35.5 \\
\hline 24 & $\begin{array}{l}\text { For manual testing job, training is required from } \\
\text { industry /private institute. }\end{array}$ & 24 & 22.4 & 40 & 37.4 & 5 & 4.7 & 21 & 19.6 & 17 & 15.9 \\
\hline 25 & In house training is required for manual testing. & 30 & 28.0 & 34 & 31.8 & 7 & 6.5 & 20 & 18.7 & 16 & 15.0 \\
\hline
\end{tabular}

Factor 6: In factor 6, one variable found to be high factor loading i.e. .653(Live projects of ST were not included in curriculum through industry collaboration). So this variable can be named as "Live projects of ST are not included in curriculum through industry collaboration".
Factor 7: In factor 7, two variables found to be high factor loading i.e. .708(ST syllabus provided knowledge of black box testing), and .528(ST syllabus provides knowledge of white box testing). So these two variables can be clubbed and factor may be named as "Software testing syllabus includes theory of manual testing techniques". 
Table-2: Responses of survey on automated testing

\begin{tabular}{|c|c|c|c|c|c|c|c|c|c|c|c|}
\hline & \multirow[t]{2}{*}{ Questions } & \multicolumn{2}{|c|}{$\begin{array}{l}\text { Strongly } \\
\text { Disagree }\end{array}$} & \multicolumn{2}{|c|}{ Disagree } & \multicolumn{2}{|c|}{ Neutral } & \multicolumn{2}{|c|}{ Agree } & \multicolumn{2}{|c|}{$\begin{array}{l}\text { Strongly } \\
\text { Agree }\end{array}$} \\
\hline & & 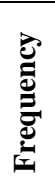 & $\%$ & 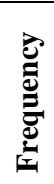 & $\%$ & 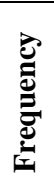 & $\%$ & 它 & $\%$ & 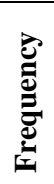 & $\%$ \\
\hline 1 & $\begin{array}{l}\text { Academic curriculum meets the requirements of } \\
\text { present } s / w \text { industry. }\end{array}$ & 14 & 13.5 & 43 & 41.3 & 30 & 28.8 & 12 & 11.5 & 5 & 4.8 \\
\hline 2 & $\begin{array}{l}\text { Academic curriculum provides basic knowledge of } \\
\text { software testing (ST). }\end{array}$ & 7 & 6.7 & 21 & 20.2 & 28 & 26.9 & 36 & 34.6 & 12 & 11.5 \\
\hline 3 & $\begin{array}{l}\text { In depth knowledge of software testing was part of } \\
\text { curriculum. }\end{array}$ & 4 & 3.8 & 25 & 24.0 & 19 & 18.3 & 42 & 40.4 & 14 & 13.5 \\
\hline 4 & $\begin{array}{l}\text { ST should be included as a separate subject in } \\
\text { academic curriculum. }\end{array}$ & 2 & 1.9 & 13 & 12.5 & 35 & 33.7 & 39 & 37.5 & 15 & 14.4 \\
\hline 5 & $\begin{array}{l}\begin{array}{l}\text { Automation testing was separate subject in } \\
\text { curriculum. }\end{array} \\
\end{array}$ & 18 & 17.3 & 46 & 44.2 & 23 & 22.1 & 15 & 14.4 & 2 & 1.9 \\
\hline 6 & $\begin{array}{l}\text { Automation testing should be part of software } \\
\text { testing. }\end{array}$ & 4 & 3.8 & 9 & 8.7 & 8 & 7.7 & 42 & 40.4 & 41 & 39.4 \\
\hline 7 & $\begin{array}{l}\text { Implementation (Practical) of software testing } \\
\text { strategies was part of curriculum. }\end{array}$ & 7 & 6.7 & 17 & 16.3 & 21 & 20.2 & 36 & 34.6 & 23 & 22.1 \\
\hline 8 & $\begin{array}{l}\text { Marks of implementation (practical's) of ST added to } \\
\text { marks of final degree. }\end{array}$ & 2 & 1.9 & 23 & 22.1 & 28 & 26.9 & 33 & 31.7 & 18 & 17.3 \\
\hline 9 & $\begin{array}{l}\text { Demo version of automated testing tools were used } \\
\text { for practical of software testing. }\end{array}$ & 14 & 13.5 & 27 & 26.0 & 29 & 27.9 & 25 & 24.0 & 9 & 8.7 \\
\hline 10 & $\begin{array}{l}\begin{array}{l}\text { Automation testing was introductory part of } \\
\text { curriculum. }\end{array} \\
\end{array}$ & 26 & 25.0 & 47 & 45.2 & 20 & 19.2 & 9 & 8.7 & 2 & 1.9 \\
\hline 11 & Automation testing was part of software testing. & 26 & 25.0 & 53 & 51.0 & 9 & 8.7 & 11 & 10.6 & 5 & 4.8 \\
\hline 12 & $\begin{array}{l}\text { ST syllabus provides knowledge of various testing } \\
\text { techniques. }\end{array}$ & 5 & 4.8 & 9 & 8.7 & 23 & 22.1 & 34 & 32.7 & 33 & 31.7 \\
\hline 13 & $\begin{array}{l}\text { Automation testing tools learned by private training } \\
\text { institute. }\end{array}$ & 12 & 11.5 & 16 & 15.4 & 10 & 9.6 & 41 & 39.4 & 25 & 24.0 \\
\hline 14 & $\begin{array}{l}\text { Automation testing was part of software engineering } \\
\text { subject. }\end{array}$ & 34 & 32.7 & 49 & 47.1 & 8 & 7.7 & 7 & 6.7 & 6 & 5.8 \\
\hline 15 & $\begin{array}{l}\text { In depth knowledge of automated testing tools was } \\
\text { part of curriculum. }\end{array}$ & 21 & 20.2 & 40 & 38.5 & 30 & 28.8 & 8 & 7.7 & 5 & 4.8 \\
\hline 16 & $\begin{array}{l}\text { Implementation (Practical) of software testing } \\
\text { strategies should be part of curriculum. }\end{array}$ & 21 & 20.2 & 53 & 51.0 & 19 & 18.3 & 7 & 6.7 & 4 & 3.8 \\
\hline 17 & $\begin{array}{l}\text { Project classes and practical viva must under control } \\
\text { of industry expert. }\end{array}$ & 27 & 26.0 & 42 & 40.4 & 13 & 12.5 & 15 & 14.4 & 7 & 6.7 \\
\hline 18 & $\begin{array}{l}\text { Live projects of automated ST should be included in } \\
\text { curriculum through industry collaboration. }\end{array}$ & 8 & 7.7 & 21 & 20.2 & 28 & 26.9 & 31 & 29.8 & 16 & 15.4 \\
\hline 19 & $\begin{array}{l}\text { Automation testing tools learned by in house training } \\
\text { by company. }\end{array}$ & 8 & 7.7 & 10 & 9.6 & 24 & 23.1 & 35 & 33.7 & 27 & 26.0 \\
\hline 20 & $\begin{array}{l}\text { Licensed tools of automated testing with full } \\
\text { functionality were part of curriculum. }\end{array}$ & 10 & 9.6 & 21 & 20.2 & 31 & 29.8 & 32 & 30.8 & 10 & 9.6 \\
\hline 21 & $\begin{array}{l}\text { Automation testing tools learned by self-study } \\
\text { /google /you tube. }\end{array}$ & 9 & 8.7 & 13 & 12.5 & 24 & 23.1 & 30 & 28.8 & 28 & 26.9 \\
\hline 22 & $\begin{array}{l}\text { Final project training is actually done at } \mathrm{s} / \mathrm{w} \text { industry } \\
\text { in testing domain }\end{array}$ & 8 & 7.7 & 20 & 19.2 & 24 & 23.1 & 35 & 33.7 & 17 & 16.3 \\
\hline 23 & $\begin{array}{l}\text { ST syllabus provides knowledge of object oriented } \\
\text { testing. }\end{array}$ & 6 & 5.8 & 8 & 7.7 & 8 & 7.7 & 50 & 48.1 & 32 & 30.8 \\
\hline 24 & $\begin{array}{l}\text { Industries provide in house training for self- } \\
\text { developed testing tools. }\end{array}$ & 4 & 3.8 & 11 & 10.6 & 10 & 9.6 & 45 & 43.3 & 34 & 32.7 \\
\hline 25 & $\begin{array}{l}\text { All automation testing tools could not be included in } \\
\text { curriculum. }\end{array}$ & 3 & 2.9 & 6 & 5.8 & 14 & 13.5 & 44 & 42.3 & 37 & 35.6 \\
\hline
\end{tabular}


Table-3: Total Variance Explained

\begin{tabular}{|c|c|c|c|c|c|c|c|c|c|}
\hline \multirow[t]{2}{*}{ Component } & \multicolumn{3}{|c|}{ Initial Eigenvalues } & \multicolumn{3}{|c|}{ Extraction Sums of Squared Loadings } & \multicolumn{3}{|c|}{ Rotation Sums of Squared Loadings } \\
\hline & Total & $\begin{array}{l}\% \text { of } \\
\text { Variance }\end{array}$ & $\begin{array}{l}\text { Cumulativ } \\
\text { e } \%\end{array}$ & Total & $\begin{array}{l}\% \text { of } \\
\text { Variance }\end{array}$ & $\begin{array}{l}\text { Cumulative } \\
\%\end{array}$ & Total & $\begin{array}{l}\% \text { of } \\
\text { Variance }\end{array}$ & $\begin{array}{l}\text { Cumulative } \\
\%\end{array}$ \\
\hline 1 & 6.185 & 24.741 & 24.741 & 6.185 & 24.741 & 24.741 & 4.871 & 19.482 & 19.482 \\
\hline 2 & 2.179 & 8.714 & 33.455 & 2.179 & 8.714 & 33.455 & 2.001 & 8.004 & 27.486 \\
\hline 3 & 1.748 & 6.993 & 40.448 & 1.748 & 6.993 & 40.448 & 1.827 & 7.307 & 34.793 \\
\hline 4 & 1.633 & 6.534 & 46.981 & 1.633 & 6.534 & 46.981 & 1.574 & 6.297 & 41.091 \\
\hline 5 & 1.458 & 5.834 & 52.815 & 1.458 & 5.834 & 52.815 & 1.556 & 6.223 & 47.314 \\
\hline 6 & 1.312 & 5.247 & 58.062 & 1.312 & 5.247 & 58.062 & 1.538 & 6.153 & 53.467 \\
\hline 7 & 1.207 & 4.828 & 62.890 & 1.207 & 4.828 & 62.890 & 1.533 & 6.131 & 59.598 \\
\hline 8 & 1.055 & 4.219 & 67.109 & 1.055 & 4.219 & 67.109 & 1.501 & 6.003 & 65.601 \\
\hline 9 & 1.021 & 4.084 & 71.192 & 1.021 & 4.084 & 71.192 & 1.398 & 5.592 & 71.192 \\
\hline 1 & .868 & 3.470 & 74.663 & & & & & & \\
\hline 11 & .836 & 3.344 & 78.007 & & & & & & \\
\hline 12 & .768 & 3.072 & 81.079 & & & & & & \\
\hline 13 & .670 & 2.682 & 83.761 & & & & & & \\
\hline 14 & .614 & 2.455 & 86.216 & & & & & & \\
\hline 15 & .585 & 2.341 & 88.557 & & & & & & \\
\hline 16 & .507 & 2.029 & 90.587 & & & & & & \\
\hline 17 & .482 & 1.930 & 92.516 & & & & & & \\
\hline 18 & .379 & 1.515 & 94.032 & & & & & & \\
\hline 19 & .322 & 1.288 & 95.320 & & & & & & \\
\hline 20 & .265 & 1.059 & 96.379 & & & & & & \\
\hline 21 & .247 & .988 & 97.367 & & & & & & \\
\hline 22 & .201 & .805 & 98.172 & & & & & & \\
\hline 23 & .184 & .735 & 98.907 & & & & & & \\
\hline 24 & .141 & .563 & 99.470 & & & & & & \\
\hline 25 & .132 & .530 & 100.000 & & & & & & \\
\hline
\end{tabular}

Extraction Method: Principal Component Analysis.

Factor 8: In factor 8, two variables found to be high factor loading i.e. .840(Academic curriculum of SE meets the requirements of present $\mathrm{s} / \mathrm{w}$ industry), and .573(SE concepts used in industry are same as you studied). So these two variables can be clubbed and factor may be named as "Academic curriculum of SE meets the requirements of present s/w industry and SE concepts used in industry are same as studied".

Factor 9: In factor 9, one variable found to be high factor loading i.e. .663(More emphasis on programming languages than SE in curriculum). So this variable can be named as "More emphasizes on programming language".

\subsection{Gap Analysis of Theory and Practice for Automated Testing}

Data of 104 respondents was analyzed by applying factor analysis using SPSS software. The purpose of factor analysis is to reduce multiple variables to a lesser number of underlying factors that are being measured by the variables. To test the appropriateness of factor analysis technique Kaiser-Meyer-Olkin (KMO) measure of sample adequacy is used. KMO compares the magnitude of observed correlation coefficients to magnitude of partial correlation coefficients. KMO identified values greater than 0.5 might desirable. In our analysis, the value of KMO comes out is .739 with significance .000 , which is acceptable.
Principal Component Analysis is a dimension reduction technique to analyze factors responsible for gap in manual software testing. A set of factors is identified based upon possible information available in the data. Seven factors are extracted on the basis of Principal Component Analysis. These seven factors describe the relationship among variables in a best way showing cumulative \%age of variances, it is observed that seven factors identifying $68.146 \%$ of variance contributed by first component is 18.391 followed by second is 30.348 , third is 39.247 , fourth is 47.957 , fifth is 55.767 , sixth is 62.924 , seventh is 68.146 [Table-5]. In rotated component matrix, a value greater than .500 is considered for analysis across these seven factors against 25 question statements.

\subsubsection{Automated Testing Factors [Table-6]}

Factor 1: In factor 1, five variables found to be high factor loading i.e. .592(Automation testing should be part of software testing), .688(Automation testing tools learned by private training institute), .690(Automation testing tools learned by in house training by company), .694(Automation testing tools learned by self-study /google /you tube), and .806(Industries provide in house training for self-developed testing tools). So these five variables can be clubbed and factor may be named as "There is gap between theoretical and actual implementation of automation s/w testing and automated testing tools are learned during job not in academic". 
Table-4: Rotated Component Matrix(Rotation converged in 16 iterations)

\begin{tabular}{|c|c|c|c|c|c|c|c|c|c|}
\hline & \multicolumn{9}{|c|}{ Component } \\
\hline & 1 & 2 & 3 & 4 & 5 & 6 & 7 & 8 & 9 \\
\hline $\begin{array}{l}\text { Academic curriculum provides only basic knowledge } \\
\text { of Software testing (ST). }\end{array}$ & -.054 & .071 & .159 & -.222 & -.325 & .350 & .421 & -.204 & .373 \\
\hline $\begin{array}{l}\text { Academic curriculum covers in depth knowledge of } \\
\text { Software engineering (SE). }\end{array}$ & .002 & -.008 & .133 & .736 & .009 & .080 & -.224 & .222 & -.025 \\
\hline $\begin{array}{l}\text { Implementation(Practicals) of software engineering } \\
\text { concepts was part of curriculum. }\end{array}$ & .056 & .007 & .847 & .120 & -.137 & -.076 & -.074 & .116 & -.128 \\
\hline $\begin{array}{l}\text { Marks of implementation (practical) of SE added to } \\
\text { marks of final degree. }\end{array}$ & .059 & -.040 & .839 & -.018 & .161 & -.063 & .080 & .051 & .164 \\
\hline $\begin{array}{l}\text { SE syllabus covers basic knowledge of software } \\
\text { testing. }\end{array}$ & -.320 & .059 & .147 & -.243 & .686 & .009 & .027 & -.045 & .061 \\
\hline $\begin{array}{l}\text { Academic curriculum of SE meets the requirements } \\
\text { of present } \mathrm{s} / \mathrm{w} \text { industry }\end{array}$ & -.070 & .132 & .099 & .087 & -.124 & -.058 & .076 & .840 & .140 \\
\hline $\begin{array}{l}\text { ST was a separate subject with focus on manual } \\
\text { testing in academic curriculum. }\end{array}$ & -.136 & .001 & -.174 & .314 & .660 & -.351 & -.007 & -.020 & -.020 \\
\hline dge of black box testing. & .264 & .040 & -.304 & .005 & .082 & -.086 & .708 & .223 & -.056 \\
\hline te box testing. & .294 & .092 & .207 & -.001 & -.026 & .020 & .528 & -.026 & -.046 \\
\hline $\begin{array}{l}\text { In depth knowledge of complete software testing was } \\
\text { part of curriculum. }\end{array}$ & -.248 & -.198 & .274 & .336 & .283 & -.131 & $\mid-.183$ & .321 & -.007 \\
\hline $\begin{array}{l}\text { Final project training is actually done at } \mathrm{s} / \mathrm{w} \text { industry } \\
\text { in testing domain }\end{array}$ & .801 & .119 & .054 & -.057 & -.250 & .089 & .002 & .001 & .059 \\
\hline $\begin{array}{l}\text { Project classes and practical viva was under control } \\
\text { of industry expert. }\end{array}$ & -.701 & .272 & -.063 & -.084 & .030 & -.056 & -.217 & .095 & .236 \\
\hline $\begin{array}{l}\text { Implementation (Practical) of software testing } \\
\text { strategies was part of curriculum. }\end{array}$ & .045 & .889 & -.126 & -.188 & -.002 & .038 & -.005 & .144 & -.028 \\
\hline $\begin{array}{l}\text { SE curriculum covers architectural design of } \\
\text { software. }\end{array}$ & -.251 & -.044 & -.035 & .708 & -.033 & -.123 & .345 & -.162 & .064 \\
\hline $\begin{array}{l}\text { Regression testing is part of theory/software } \\
\text { maintenance. }\end{array}$ & -.1 & -.007 & .129 & -.124 & .152 & -.786 & .210 & -.102 & -.098 \\
\hline $\begin{array}{l}\text { Manual s/w testing concepts used in industry are } \\
\text { same as you studied. }\end{array}$ & .505 & .460 & .068 & .131 & -.055 & .272 & .147 & -.209 & -.219 \\
\hline $\begin{array}{l}\text { More emphasis on programming languages than SE } \\
\text { in curriculum }\end{array}$ & .287 & .236 & .028 & .130 & -.122 & .030 & -.290 & .084 & .663 \\
\hline $\begin{array}{l}\text { Live projects of ST were included in curriculum } \\
\text { through industry collaboration. }\end{array}$ & .235 & .186 & -.080 & -.168 & -.010 & .653 & .204 & -.196 & -.005 \\
\hline $\begin{array}{l}\text { Implementation (Practical) of software testing } \\
\text { strategies should be part of curriculum. }\end{array}$ & -.883 & -.129 & .050 & .057 & .113 & -.116 & -.073 & .067 & -.035 \\
\hline $\begin{array}{l}\text { Marks of implementation (practical's) of ST added to } \\
\text { marks of final degree. }\end{array}$ & .395 & .666 & .128 & .141 & .033 & .148 & .200 & -.131 & -.021 \\
\hline $\begin{array}{l}\text { Able to join software testing job directly after } \\
\text { completion of academics. }\end{array}$ & -.019 & .398 & -.004 & .043 & -.122 & -.081 & -.118 & -.040 & -.708 \\
\hline $\begin{array}{l}\text { ST syllabus provides knowledge of object oriented } \\
\text { testing. }\end{array}$ & -.696 & -.109 & -.055 & .156 & .253 & .122 & -.075 & -.071 & -.261 \\
\hline SE concepts used in industry are same as you studied. & -.274 & -.270 & .128 & -.003 & .442 & .040 & .047 & .573 & -.185 \\
\hline $\begin{array}{l}\text { For manual testing job, training is required from } \\
\text { industry/private institute. }\end{array}$ & .832 & .161 & -.010 & -.067 & -.044 & .128 & .222 & -.035 & .078 \\
\hline In house training is required for manual testing . & .847 & .113 & .016 & -.103 & .012 & .166 & .001 & -.138 & .118 \\
\hline
\end{tabular}

Extraction Method: Principal Component Analysis. Rotation Method: Varimax with Kaiser Normalization.

Factor 2: In factor 2, four variables found to be high factor loading i.e. .808 (Demo version of automated testing tools were not used for practical of software testing), .822(Live projects of automated ST were not included in curriculum through industry collaboration), .858(Licensed tools of automated testing with full functionality were not part of curriculum), and .772(Final project training is actually done at $\mathrm{s} / \mathrm{w}$ industry in testing domain). So these four variables can be clubbed and factor may be named as "Lack of practical of automated s/w testing in curriculum".

Factor 3: In factor 3, two variables found to be high factor loading i.e. .675(Implementation (Practical) of software testing strategies was part of curriculum), and .795(Marks of implementation (practical's) of ST added to marks of final degree). So these two variables can be clubbed and factor may be named as "Software testing syllabus includes practical of testing techniques and marks are added to degree".

Factor 4: In factor 4, two variables found to be high factor loading i.e. .825(Academic curriculum provides basic knowledge of software testing), and .718(In depth knowledge of software testing was part of curriculum). So these two variables can be clubbed and factor may be named as "Software testing has theoretical foundation in curriculum". 
Table-5: Total Variance Explained

\begin{tabular}{|c|c|c|c|c|c|c|c|c|c|}
\hline \multirow[t]{2}{*}{ Component } & \multicolumn{3}{|c|}{ Initial Eigenvalues } & \multicolumn{3}{|c|}{$\begin{array}{llll}\begin{array}{l}\text { Extraction Sums of } \\
\text { Loadings }\end{array} & & & \\
\end{array}$} & \multicolumn{3}{|c|}{ Rotation Sums of Squared Loadings } \\
\hline & Total & $\begin{array}{l}\% \text { of } \\
\text { Variance }\end{array}$ & $\begin{array}{l}\text { Cumulative } \\
\%\end{array}$ & Total & $\begin{array}{l}\% \text { of } \\
\text { Variance }\end{array}$ & $\begin{array}{l}\text { Cumulative } \\
\%\end{array}$ & Total & $\begin{array}{l}\% \text { of } \\
\text { Variance }\end{array}$ & $\begin{array}{l}\text { Cumulative } \\
\%\end{array}$ \\
\hline 1 & 7.307 & 29.229 & 29.229 & 7.307 & 29.229 & 29.229 & 4.598 & 18.391 & 18.391 \\
\hline 2 & 2.562 & 10.250 & 39.479 & 2.562 & 10.250 & 39.479 & 2.989 & 11.957 & 30.348 \\
\hline 3 & 2.187 & 8.749 & 48.228 & 2.187 & 8.749 & 48.228 & 2.225 & 8.899 & 39.247 \\
\hline 4 & 1.474 & 5.895 & 54.122 & 1.474 & 5.895 & 54.122 & 2.177 & 8.710 & 47.957 \\
\hline 5 & 1.253 & 5.011 & 59.133 & 1.253 & 5.011 & 59.133 & 1.952 & 7.810 & 55.767 \\
\hline 6 & 1.177 & 4.707 & 63.840 & 1.177 & 4.707 & 63.840 & 1.789 & 7.157 & 62.924 \\
\hline 7 & 1.076 & 4.305 & 68.146 & 1.076 & 4.305 & 68.146 & 1.305 & 5.222 & 68.146 \\
\hline 8 & .923 & 3.693 & 71.839 & & & & & & \\
\hline 9 & .874 & 3.495 & 75.334 & & & & & & \\
\hline 10 & .728 & 2.910 & 78.244 & & & & & & \\
\hline 11 & .669 & 2.676 & 80.920 & & & & & & \\
\hline 12 & .616 & 2.463 & 83.383 & & & & & & \\
\hline 13 & .601 & 2.405 & 85.789 & & & & & & \\
\hline 14 & .500 & 1.998 & 87.787 & & & & & & \\
\hline 15 & .473 & 1.890 & 89.677 & & & & & & \\
\hline 16 & .423 & 1.692 & 91.369 & & & & & & \\
\hline 17 & .353 & 1.414 & 92.783 & & & & & & \\
\hline 18 & .337 & 1.350 & 94.133 & & & & & & \\
\hline 19 & .308 & 1.232 & 95.365 & & & & & & \\
\hline 20 & .295 & 1.179 & 96.544 & & & & & & \\
\hline 21 & .258 & 1.033 & 97.577 & & & & & & \\
\hline 22 & .213 & .850 & 98.427 & & & & & & \\
\hline 23 & .172 & .689 & 99.116 & & & & & & \\
\hline 24 & .141 & .563 & 99.679 & & & & & & \\
\hline 25 & .080 & .321 & 100.000 & & & & & & \\
\hline
\end{tabular}

Extraction Method: Principal Component Analysis.

Factor 5: In factor 5, three variables found to be high factor loading i.e. ..624(Automation testing was not separate subject in curriculum), .789(Automation testing was introductory part of curriculum), and .554(In depth knowledge of automated testing tools was not part of curriculum). So these three variables can be clubbed and factor may be named as "Lack of theory of automated s/w testing in curriculum".

Factor 6: In factor 6, two variables found to be high factor loading i.e. 804 (ST syllabus provides knowledge of various testing techniques), and .553(ST syllabus provides knowledge of object oriented testing). So these two variables can be clubbed and factor may be named as "Software testing syllabus includes theory of testing techniques".

Factor 7: In factor 7, one variable found to be high factor loading i.e. .628(Academic curriculum doesn't meet the requirements of present $\mathrm{s} / \mathrm{w}$ industry). So this variable can be named as "Academic curriculum of CS/IT courses doesn't meet the requirement of industry".

\section{CONCLUSION AND RECOMMENDATION}

The findings presented in this paper were drawn from the responses of two surveys: 107 respondents for manual testing and 104 respondents for automated testing from the Indian IT industry. We believe that the findings are general in nature with actions required from academia and industry.

For manual testing nine factors are identified viz.

There is no gap between theoretical and actual implementation of manual s/w testing;

Software testing syllabus includes practical of manual testing techniques and marks are added to degree;

Software engineering practical are not adequate in curriculum but marks are added to degree;

Software engineering has theoretical foundation in curriculum; Software testing has theoretical foundation in curriculum;

Live projects of ST are not included in curriculum through industry collaboration;

Software testing syllabus includes theory of manual testing techniques; 
Table-6: Rotated Component Matrix (Rotation converged in 11 iterations)

\begin{tabular}{|c|c|c|c|c|c|c|c|}
\hline & \multicolumn{7}{|c|}{ Component } \\
\hline & 1 & 2 & 3 & 4 & 5 & 6 & 7 \\
\hline Academic curriculum meets the requirements of present s/w industry. & -.202 & .014 & .278 & .103 & .022 & -.106 & .628 \\
\hline $\begin{array}{l}\text { Academic curriculum provides basic knowledge of software testing } \\
\text { (ST). }\end{array}$ & -.254 & .034 & -.212 & .825 & .094 & .011 & -.078 \\
\hline In depth knowledge of software testing was part of curriculum. & -.172 & .195 & -.116 & .718 & .199 & -.022 & .244 \\
\hline ST should be included as a separate subject in academic curriculum. & .201 & -.191 & -.522 & .216 & -.125 & .244 & .380 \\
\hline Automation testing was separate subject in curriculum. & .115 & -.166 & -.034 & .408 & .624 & -.126 & -.071 \\
\hline Automation testing should be part of software testing. & .592 & -.038 & -.291 & -.182 & .032 & .051 & .447 \\
\hline $\begin{array}{l}\text { Implementation (Practical) of software testing strategies was part of } \\
\text { curriculum. }\end{array}$ & -.293 & .042 & .675 & -.185 & .105 & -.038 & .024 \\
\hline $\begin{array}{l}\text { Marks of implementation (practical's) of ST added to marks of final } \\
\text { degree. }\end{array}$ & -.094 & .097 & .795 & -.028 & .066 & .105 & .205 \\
\hline $\begin{array}{l}\text { Demo version of automated testing tools were used for practical of } \\
\text { software testing. }\end{array}$ & .080 & .808 & .033 & -.132 & .070 & .356 & .060 \\
\hline Automation testing was introductory part of curriculum. & -.291 & .229 & .069 & -.090 & .789 & .152 & .130 \\
\hline Automation testing was part of software testing. & -.565 & .024 & .301 & .323 & .072 & .159 & - \\
\hline ST syllabus provides knowledge of various testing techniques. & .247 & -.015 & -.016 & -.035 & -.042 & .804 & \\
\hline Automation testing tools learned by private training institute. & .688 & -.097 & -.278 & -.223 & -.170 & .174 & .012 \\
\hline Automation testing was part of software engineering subject. & -.185 & .133 & .447 & .484 & -.074 & -.340 & .114 \\
\hline In depth knowledge of automated testing tools was part of curriculum. & -.296 & .188 & .248 & .095 & .554 & -.147 & -.230 \\
\hline $\begin{array}{l}\text { Implementation (Practical) of software testing strategies should be } \\
\text { part of curriculum. }\end{array}$ & -.682 & .074 & .245 & .096 & .184 & -.068 & .080 \\
\hline $\begin{array}{l}\text { Project classes and practical viva must under control of industry } \\
\text { expert. }\end{array}$ & -.644 & .183 & .184 & .309 & .230 & -.311 & .105 \\
\hline $\begin{array}{l}\text { Live projects of automated ST were included in curriculum through } \\
\text { industry collaboration. }\end{array}$ & -.099 & .822 & .096 & .226 & .141 & -.053 & -.092 \\
\hline Automation testing tools learned by in house training by company. & .690 & -.077 & -.140 & -.080 & .200 & .308 & -.019 \\
\hline $\begin{array}{l}\text { Licensed tools of automated testing with full functionality were part } \\
\text { of curriculum. }\end{array}$ & -.078 & .858 & -.005 & .053 & -.028 & -.113 & .077 \\
\hline Automation testing tools learned by self-study /google /you tube. & .694 & -.188 & .057 & -.090 & -.072 & .118 & -388 \\
\hline $\begin{array}{l}\text { Final project training is actually done at } \mathrm{s} / \mathrm{w} \text { industry in testing } \\
\text { domain }\end{array}$ & -.294 & .772 & .237 & .100 & .104 & -.245 & -.030 \\
\hline ST syllabus provides knowledge of object oriented testing. & .543 & -.046 & .052 & .005 & -.262 & .553 & - \\
\hline Industries provide in house training for self-developed testing tools. & .806 & -.015 & -.020 & .030 & -.148 & .109 & .066 \\
\hline All automation testing tools could not be included in curriculum. & .115 & -.122 & -.149 & -.286 & -.502 & .306 & - \\
\hline
\end{tabular}

Extraction Method: Principal Component Analysis. Rotation Method: Varimax with Kaiser Normalization.

Academic curriculum of $\mathrm{SE}$ meets the requirements of present s/w industry and SE concepts used in industry are same as studied;

More emphasizes on programming language.

The conclusion of all these is that there is no gap between theory and practices of manual testing.

For automated testing, seven factors are identified viz.

There is gap between theoretical and actual implementation of automation s/w testing and automated testing tools are learned during job not in academic;

Lack of practical of automated s/w testing in curriculum;

Software testing syllabus includes practical of testing techniques and marks are added to degree;

Software testing has theoretical foundation in curriculum;

Lack of theory of automated s/w testing in curriculum;
Software testing syllabus includes theory of testing techniques;

Academic curriculum of CS/IT courses doesn't meet the requirement of industry.

The conclusion of all these is that there is gap between theory and practices of automated testing.

It is recommended that collaboration between the industry and academia including faculty training and curriculum development is crucial to bridge this gap. Students must be exposed to testing tools that are standard in the software industry. They need to acquire more practical testing experience and work on real-life projects that will permit them to acquire technical, soft and IT offshore outsourcing skills. 


\section{REFERENCES}

[1] S.P. Ng, T. Murnane, K. Reed, D. Grant, T.Y., Chen A, 2004. Preliminary Survey on Software Testing Practices in Australia, Proceedings of the 2004 Australian Software Engineering Conference (ASWEC'04).

[2] Emelie Engstr"om and Per Runeson, 2010. A Qualitative Survey of Regression Testing Practices, LNCS 6156, Springer-Verlag pp. 3-16.

[3] Vidya Kulkarni, Christelle Scharff, et. al 2010. From Student to Software Engineer in the Indian IT Industry: A Survey of Training, 23rd IEEE Conference on Software Engineering Education and Training.

[4] Causevic, A., Sundmark, D., Punnekkat, S, 2010. An industrial survey on contemporary aspects of software testing, Proceedings of the 3rd International Conference on Software Testing Verification and Validation, pp. 393-401.

[5] Rooksby, J., Rouncefield, M., Sommerville,I, 2009. Testing in the wild: The social and organisational dimensions of real world practice, Computer Supported Cooperative Work (CSCW) 18(5), pp 559580.
[6] Grindal, M., Offutt, J., Mellin, J, 2006. On the testing maturity of software producing organizations. In: Testing: Academia \& Industry Conference-Practice And Research Techniques, TAIC/PART.

[7] Murnane and K. Reed, 2001. On the Effectiveness of Mutation Analysis as a Black-box Testing Technique, Proceedings of the 2001 Australian Software Engineering Conference, Canberra, Australia, pp 1220.

[8] Paul C Jorgensen, 2013. Software Testing: A craftsman's Approach. CRC Press.

[9] R. S. Pressman, 2004. Software Engineering: A Practitioners Approach, McGraw-Hill, International Edition.

[10] K. Reed, 2002. Testing, testing, testing, Australian Computer Society Information Age, pp 56-58.

[11] L. Groves, R. Nickson, G. Reeves, et. al 2000. A Survey of Software Practices in the New Zealand Software Industry", Proceedings of the Australian Software Engineering Conference, pp 189-101.

[12] IEEE: IEEE standard for software test documentation. IEEE Std(829-1983, Revision) (1998) 